\title{
Informationen der ÄMKA
}

Manuelle Medizin 2018 · 56:212

https://doi.org/10.1007/s00337-018-

0389-2

(c) Springer Medizin Verlag $\mathrm{GmbH}$, ein

Teil von Springer Nature 2018

Ärztegesellschaft für Manuelle Kinderbehandlung

\& Atlastherapie e.V.

\section{Ärztegesellschaft für Manuelle Kinderbehandlung} und Atlastherapie (ÄMKA) e.V.

in Kooperation mit dem Dr. Karl-Sell-Ärzteseminar Neutrauchburg (MWE) e.V.

Anna-von-Borries-Str. 1-7

30625 Hannover

Fax: 0511-5354675

info@aegamk.de

www.aegamk.de
V.i.S.d.P.:

Ruth Kamping

Scheidestr. 3

30625 Hannover

\section{Kursprogramm Hannover}

\author{
Kursort Annastift Hannover \\ Kursleiter R. Kamping, Dr. A. Thren, Dr. P. Weng
}

\section{8}

Kinderkurs D

Atlaskurs Teil I

19.-21.04.18

Kinderkurs A

12. -13.09 .18$

Atlaskurs Teil II

14. -15.09 .18$

28. -29.11 .18$

Kinderkurs B

30.11.-01.12.18

Anmeldung ausschließlich über:

Diakonische Dienste Hannover GmbH

Akademie für Fort- und Weiterbildung

Anna-von-Borries-Str. 1-7, 30625 Hannover

Info-Tel: 0511-5354662, Fax: 0511-5354672

akademie@diakovere.de,www.ddh-akademie.de

\section{Kursprogramm Isny- Neutrauchburg}

In Isny finden 2018 keine Kinderkurse statt. Eine neue Kursserie beginnt im Februar 2019.

\section{Atlaskurs 1}

Besondere Bedeutung des zervikookzipitalen Überganges (Kopfgelenke 1), praktische Übungen.

\section{Atlaskurs 2}

Kopfgelenk (Teil 2), klinische und physiologische Aspekte, therapeutischer Ansatz, Simulator-Training. Schriftl. Test.

\section{Kinder-Kurs A Diagnostikkurs 1}

Neurophysiologische und manualmedizinische Untersuchung im Säuglingsalter: frühkindliche Entwicklung, Reflexologie, sensomotorische Integration, funktionelle und segmentale Befundung der sensorischen Schlüsselregionen.

\section{Kinder-Kurs B Diagnostikkurs 2}

Sensomotorische Dysfunktion im Kindesalter, ADS, Therapiegrundsätze bei idiopathischer Skoliose. Infantile Zerebralparese: Propädeutik und Untersuchungstechnik.

\section{Kinder-Kurs C Therapiekurs 1}

Manuelle Impuls - und Weichteiltechniken bei Säuglingen und Kindern an Wirbelsäule und Extremitäten.

\section{Kinder-Kurs D Therapiekurs 2}

Manuelle Impuls- und Weichteiltechniken an Wirbelsäule und Extremitäten (Teil 2), Atlastherapie bei Säuglingen und Kindern.

Examen. Zertifikat

Die Atlaskurse können auch unabhängig von den Kinderkursen besucht werden. Ein kombinierter Besuch ist jedoch sinnvoll, da die Atlaskurse Voraussetzung und wichtiger Bestandteil der Ausbildung in manueller Kinderbehandlung sind. So sind die Atlaskurse den Kinderkursen $\mathrm{A}+\mathrm{B}$ vorangestellt.

Kursvoraussetzung ist die Approbation und die abgeschlossene Ausbildung in Manueller Medizin. 